\title{
Ki-67 immunostaining in astrocytomas: Association with histopathological grade - A South Indian study
}

\author{
Nandish Vastrad Shivaprasad, Suchitha Satish, Sunila Ravishankar, Manjunath Gubbi Vimalambike \\ Department of Pathology, JSS Medical College, JSS University, Mysore, Karnataka, India
}

\section{ABSTRACT}

Background: Astrocytomas are the most common primary tumor of the central nervous system. The distinction between different tumor grades can be tested despite criteria given by the World Health Organization (WHO). Ki-67 is a potent biological marker used in grading of astrocytomas, which estimates growth of the neoplasm quantitatively and will help in predicting prognosis accurately. Objectives: The aim of this was to study the proliferative activity using Ki-67 immunostaining and to assess the relationship of Ki-67 staining with the histopathological grading of astrocytomas. Patients and Methods: Thirty cases of histologically proven astrocytomas were studied. The histopathological grade was assessed using the 2007 WHO criteria. Immunohistochemistry for Ki-67 was done on paraffin-embedded wax sections. $P<0.05$ was considered statistically significant. Results: Thirty cases of astrocytomas studied showed a male preponderance $(\mathrm{M}: \mathrm{F}=1.72: 1)$ with a mean age of 48.1 years. Of these, Grade I, $(n=1,3.33 \%)$, Grade II, $(n=7,23.3 \%)$, Grade III $(n=6,20 \%)$, and Grade IV $(n=16,53.3 \%)$ astrocytomas were analyzed. The mean Ki-67 labeling index (LI) in Grades I, II, III, and IV was 0.02, 0.81, 9.14, and 17.81, respectively. Statistically significant difference was seen in the Ki-67 LI of low-grade (Grade II) and high-grade astrocytomas (Grades III and IV). There was concordance between histopathological grading and Ki-67 LI in 27 (90\%) and discordance in 3 (10\%) cases. Conclusion: Ki-67 LI varies considerably in different grades of astrocytomas and considerable overlaps can be observed between them. It can be of great help in situations where there is a lack of correlation between clinical parameters and histopathological diagnosis. Determination of Ki-67 LI should constitute a part of routine investigations in patients with astrocytomas.

Key words: Astrocytomas, histopathological grading, Ki-67 labeling index

\section{Introduction}

Astrocytomas are the most common primary tumor in the central nervous system (CNS). ${ }^{[1]}$ It constitutes a wide range of neoplasms that differ in their location, age distribution, growth potential, extent of invasiveness, and tendency for progression. ${ }^{[2]}$

The diagnosis is primarily based on histopathological criteria defined by the World Health Organization

\section{Address for correspondence:}

Dr. Suchitha Satish, \#892, I Block, $1^{\text {st }}$ Cross,

Ramakrishnanagar, Mysore - 570 022, Karnataka, India.

E-mail: satishsuchitha@gmail.com

\begin{tabular}{|l|l|}
\hline \multicolumn{2}{|c|}{ Access this article online } \\
\hline Quick Response Code: & Website: \\
\hline & www.ruralneuropractice.com \\
\cline { 2 - 2 } & \\
\hline & \\
\hline
\end{tabular}

(WHO) that grades astrocytomas as pilocytic astrocytoma (Grade I), diffuse astrocytoma (Grade II), anaplastic astrocytoma (Grade III), and glioblastoma (Grade IV). ${ }^{[3]}$

The histopathological examination still serves as the gold standard for making the diagnosis of astrocytoma and is thus fundamental for prognostication and therapeutic management of these patients. However, the current classification scheme is not optimal because of several parameters including tumor heterogeneity, sampling errors, poor reproducibility, and those tumors with common histology that can be genetically different. ${ }^{[1]}$

This is an open access article distributed under the terms of the Creative Commons Attribution-NonCommercial-ShareAlike 3.0 License, which allows others to remix, tweak, and build upon the work non-commercially, as long as the author is credited and the new creations are licensed under the identical terms.

For reprints contact: reprints@medknow.com

How to cite this article: Shivaprasad NV, Satish S, Ravishankar S, Vimalambike MG. Ki-67 immunostaining in astrocytomas: Association with histopathological grade - A South Indian study. J Neurosci Rural Pract 2016;7:510-4. 
Measurement of proliferative activity is important in determining the grade, recurrence span, and malignancy of astrocytoma. For these reasons, additional markers are needed to improve the diagnostic and prognostic accuracy. $\cdot{ }^{[3,4]}$

Ki-67 is a nuclear marker used to demonstrate the proliferative phase of cell cycle. It serves as an important supplemental tool in the diagnostic and prognostic evaluations of human astrocytomas and also to plan the adjuvant therapy. However, due to a greater spread of values between the various grades of astrocytoma, Ki-67 alone cannot be used as a diagnostic factor but should be used in combination with established criteria of histological malignancy. ${ }^{[2]}$ Histopathological grading may, however, over- or under-rate the actual biological behavior of astrocytomas. Thus, immunohistochemical evaluation may prove to be a vital supplementary tool for diagnostic and prognostic purposes. ${ }^{[5]}$

Although distinction between astrocytomas with a good prognosis and astrocytomas with a poor prognosis can often be made on histological features, for some tumors, histological differentiation is not clear, especially when only small fragments of tissue from stereotactically guided needle biopsies are available. It is for this reason that more objective criteria for predicting the prognosis of brain tumors are required. In recent years, the value of antibodies to proteins expressed in proliferating cells, such as Ki-67, has been investigated in astrocytic tumors. ${ }^{[1]}$

In this study, we studied the distribution of Ki-67 labeling index (LI) in various astrocytomas and correlated with the different WHO grades in a South Indian tertiary care center.

\section{Patients and Methods}

This study included thirty patients with astrocytic tumors of varying grades. Ethical clearance from the Institutional Ethical Committee was obtained. Surgically removed brain tumor tissues were fixed in formalin followed by paraffin embedding and staining with hematoxylin and eosin ( $\mathrm{H}$ and $\mathrm{E}$ ). All the cases were reviewed appropriately and graded using the WHO criteria published in 2007.

Antigen retrieval was done using sodium citrate buffer (0.01 M; pH, 6.0). Monoclonal antibody Ki-67 was used for Ki-67 antigen detection by the standard streptavidin-biotin technique (biogenix). A section from a reactive lymph node was taken as a positive control whereas sections treated with Tris buffer solution instead of primary antibody were used as negative control.

Brown granular nuclear reactivity was taken as positive. Immunostaining was evaluated in the fields consisting of regions of the tumor having the greatest number of immunoreactive cells as assessed qualitatively at the low-power examination. Where an uneven distribution of immunohistochemical labeling was evident, fields from the area of maximal labeling were chosen for counting. In glioblastomas, areas of sections free from necrosis or capillary endothelial proliferation were selected. The Ki-67 LI was the number of Ki-67-labeled tumor nuclei expressed as a percentage of a total number of tumor nuclei counted using high-power objective of the microscope $(\times 400)$. A total of at least 1000 tumor nuclei were counted in each case.

\section{Statistical analysis}

Data were analyzed using statistical software package SPSS Inc. Released 2007. SPSS for Windows, Version 16.0. Chicago, SPSS Inc. One-way ANOVA was employed to examine the correlation of $\mathrm{Ki}-67$ as a continuous variable with tumor grade and correlation of Ki-67 as a categorical variable was determined by Chi-square test. Data were expressed as mean. $P<0.05$ was considered statistically significant.

\section{Results}

In the present study, thirty cases of astrocytomas with ages ranging from 6 years to 75 years (mean age of 48.83 years) were studied. Males were affected predominantly with a male to female ratio of 1.72:1.

Among the 30 cases, $11(36.7 \%)$ were located in the right frontal lobe, $6(20 \%)$ in the left frontal lobe, $3(10 \%)$ in the right parietal lobe, $3(10 \%)$ in the left temporoparietal lobe, $2(6.7 \%)$ in the right temporoparietal lobe, and $1(3.3 \%)$ each in the fourth ventricle, left parietal, right frontoparietal, parieto-occipital, and cerebellar lobes.

The most common histological grade of astrocytic tumors in the present study was Grade IV - 16 cases (53.3\%) followed by Grade II - 7 cases (23.3\%). Grade I and Grade III constituted 3.3\% (one case) and 20\% (six cases), respectively. The results with regard to Ki-67 LI of varying grades are summarized in Table 1.

The Ki-67 LI increased with increase in the grade of the tumor. The mean Ki-67 LI of Grade II [Figure 1] was significantly lower when compared to Grade III [Figure 2] $(P<0.001)$ and Grade IV [Figure 3] $(P<0.000)$. 
The difference of mean in Ki-67 LI of Grade III was not significantly lower when compared to Grade IV. Considerable overlap was noted in Ki-67 LI across all grades of tumors. However, the differences among the means of each group were statistically significant $(P<0.001)$ [Tables 2-4]. Ki-67 LI did not vary significantly with respect to the age and gender of the individual.

Three cases of astrocytomas graded immunohistochemically did not show concordance with histopathological grade.

\section{Discussion}

Astrocytic tumors have an inherited tendency to progress and recur. The accurate grading of astrocytic tumors is of prime importance because it is critical to the patient management and survival/outcome. ${ }^{[6]}$ Although internationally accepted WHO grading system of CNS tumors is based on the histological features of $\mathrm{H}$ and $\mathrm{E}$ stained sections, there are cases where differentiation between Grade II and Grade III is difficult particularly

Table 1: Ki-67 labeling index in various grades of astrocytomas

\begin{tabular}{lccc}
\hline Tumor grade & Cases & Mean (SD) & Median \\
\hline Grade I & 1 & 0.02 & - \\
Grade II & 7 & $0.81(0.90)$ & 0.50 \\
Grade III & 6 & $9.14(5.13)$ & 9.84 \\
Grade IV & 16 & $17.81(10.73)$ & 14.27 \\
\hline SD: Standard deviation & & &
\end{tabular}

Table 2: Distribution of Ki-67 labeling index in astrocytomas of various grades

\begin{tabular}{lcccccc}
\hline Tumor grade & $\mathbf{0 - 2 . 0}$ & $\mathbf{2 . 0 1 - 5}$ & $\mathbf{7 . 5 1 - 1 0 . 0}$ & $\mathbf{1 0 . 0 1 - 2 0 . 0}$ & $\mathbf{2 0 . 0 1}$ & Total \\
\hline Grade I & 1 & 0 & 0 & 0 & 0 & 1 \\
Grade II & 6 & 1 & 0 & 0 & 0 & 7 \\
Grade III & 0 & 2 & 1 & 3 & 0 & 6 \\
Grade IV & 0 & 2 & 3 & 5 & 6 & 16 \\
\hline
\end{tabular}

There was no astrocytic tumor with Ki-67 LI between 5.01 and 7.50 . LI: Labeling index

Table 3: Comparison of Ki-67 labeling index between Grades II and III

\begin{tabular}{lccc}
\hline Grade & Cases & Mean (SD) & $\boldsymbol{P}$ \\
\hline Grade II & 7 & $0.81(0.9)$ & 0.001 \\
Grade III & 6 & $9.14(5.13)$ & \\
\hline
\end{tabular}

SD: Standard deviation

Table 4: Comparison of Ki-67 labeling index between Grades II and IV

\begin{tabular}{lccc}
\hline Grade & Cases & Mean (SD) & $\boldsymbol{P}$ \\
\hline Grade II & 7 & $0.81(0.9)$ & 0.000 \\
Grade IV & 16 & $17.81(10.73)$ & \\
\hline
\end{tabular}

SD: Standard deviation when the biopsy is small. The proliferative index derived from Ki-67 immunostaining has been found to be useful in the distinction between various grades of malignancy. ${ }^{[2]}$

Pilocytic astrocytomas (Grade I) have distinct clinical, pathological, and prognostic characteristics when compared to diffuse astrocytomas (Grade II). ${ }^{[2]}$ In the present study, there was a single case of pilocytic astrocytoma located in the cerebellum and the Ki-67 LI was 0.02 . A very low LI for pilocytic astrocytomas was noted and also no significant difference in LI between the pilocytic astrocytomas and diffuse astrocytomas was seen. ${ }^{[2,7,8]}$ No significant prognostic role has been observed for Ki-67 LI in pilocytic astrocytomas, and there is a limited role for $\mathrm{Ki}-67 \mathrm{LI}$ in determining the diagnosis in pilocytic astrocytoma. ${ }^{[2]}$

Most of the studies have found statistically significant differences in Ki-67 LI between low-grade (Grade II) and high-grade (Grades III and IV) astrocytomas. ${ }^{[4]}$ Although some studies have found a significant difference in Ki-67 LI between Grade III and Grade IV astrocytomas, others could not find a significant difference between them [Table 5]. ${ }^{[9-16]}$

Gemistocytic astrocytomas, though classified as Grade II tumors, generally show an aggressive behavior and are characterized by a low proliferative activity. The Ki-67 labeling is restricted to the small astrocytic cells. Gemistocytes lack proliferative activity, possibly indicating terminal differentiation, while small cells are the proliferating cells and their overall percentage may reflect the biological aggressiveness of these tumors. ${ }^{[17]}$ Ki-67 LI helps to identify gemistocytic astrocytomas of higher grade undergoing malignant progression. There was one case of gemistocytic astrocytomas, which was graded as II on histopathological examination, but turned out to be Grade III with a Ki-67 LI of 12.31 on immunohistochemistry (IHC). Therefore, it appears that gemistocytic astrocytomas should not be uniformly graded as Grade II but should be subdivided into Grades II and III neoplasms based on histological features and Ki-67 LI. ${ }^{[17]}$

In the present study, anaplastic astrocytoma (Grade III) showed a mean Ki-67 LI of $6.58 \%$, which included one case of anaplastic oligoastrocytoma (Grade III) with Ki-67 LI of $16.25 \%$. Some histologically defined Grade II oligoastrocytomas do not fulfill the WHO criteria for Grade III oligoastrocy tomas but display some histological features that are worrisome and seem more "atypical" than the ones seen in low-grade tumors. Oligoastrocytomas with intermediate grading features present a challenging issue 
Table 5: Comparison of mean Ki-67 labeling index with various studies

\begin{tabular}{|c|c|c|}
\hline Authors & Number of cases & Mean KI-67 LI \\
\hline \multirow[t]{3}{*}{ Ralte et al. ${ }^{[9]}$} & Grade II - 30 & Grade II -3.73 \\
\hline & Grade III - 11 & Grade III - 9.65 \\
\hline & Grade IV - 15 & Grade IV - 10.33 \\
\hline \multirow[t]{3}{*}{ Torp $^{[10]}$} & Grade II - 22 & Grade II - 2.7 \\
\hline & Grade III - 10 & Grade III - 13.9 \\
\hline & Grade IV - 9 & Grade IV - 12.1 \\
\hline \multirow[t]{3}{*}{ Neder et al. ${ }^{[11]}$} & Grade II - 10 & Grade II - 2.35 \\
\hline & Grade III - 5 & Grade III - 6.44 \\
\hline & Grade IV - 25 & Grade IV - 12.28 \\
\hline \multirow[t]{3}{*}{ Karamitopoulou et al. ${ }^{[12]}$} & Grade II - 24 & Grade II - 2.03 \\
\hline & Grade III - 26 & Grade III - 12.8 \\
\hline & Grade IV - 9 & Grade IV - 14.57 \\
\hline \multirow[t]{3}{*}{ Khalid et al. ${ }^{[13]}$} & Grade II - 24 & Grade II - 1.78 \\
\hline & Grade III - 20 & Grade III - 13.47 \\
\hline & Grade IV - 33 & Grade IV - 15.69 \\
\hline \multirow[t]{3}{*}{ Wakimoto et al. ${ }^{[14]}$} & Grade II - 19 & Grade II - 3.8 \\
\hline & Grade III - 25 & Grade III - 18.4 \\
\hline & Grade IV - 28 & Grade IV - 31.6 \\
\hline \multirow[t]{3}{*}{ Di et al. ${ }^{[15]}$} & Grade II - 29 & Grade II - 1.2 \\
\hline & Grade III - 25 & Grade III - 9 \\
\hline & Grade IV - 24 & Grade IV - 12 \\
\hline \multirow[t]{3}{*}{ Hsu et al..$^{[16]}$} & Grade II - 16 & Grade II - 0.88 \\
\hline & Grade III - 32 & Grade III - 8.75 \\
\hline & Grade IV - 33 & Grade IV - 9.12 \\
\hline \multirow[t]{3}{*}{ Present study } & Grade II - 7 & Grade II - 0.81 \\
\hline & Grade III - 6 & Grade III - 9.14 \\
\hline & Grade IV - 16 & Grade IV - 17.81 \\
\hline
\end{tabular}

LI: Labeling index

for the neuropathologist and neuro-oncologist because of uncertainty in their natural history and biological activity. Quantitation of cellular proliferation has been shown to relate to the length of survival in patients with "pure" oligodendrogliomas and astrocytomas, though proliferation does not correlate with tumor invasion. However, the Ki-67 LI does correlate with tumor grade. ${ }^{[18]}$

The mean Ki-67 LI of Grade IV tumors was $17.81 \%$. Some cases of glioblastoma had LI as low as Grade II tumors, indicating that Ki-67 LI alone cannot be used as a diagnostic measure. We had two cases of gliosarcoma with Ki-67 LI of $11.8 \%$ and $4.8 \%$. Furthermore, there was one case of small cell glioblastoma which had a highly monomorphic cell population. The Ki-67 LI was $20 \%$. Small cell glioblastoma is an aggressive histologic variant that behaves like primary glioblastoma multiforme, even in the absence of endothelial hyperplasia and necrosis. Despite considerable morphologic overlap with oligodendroglioma, especially the anaplastic variant, the clinicopathological and genetic features of this tumor are distinct. Ki-67 plays an important role in recognizing these high-grade tumors which may appear innocuous on histopathology. ${ }^{[19]}$

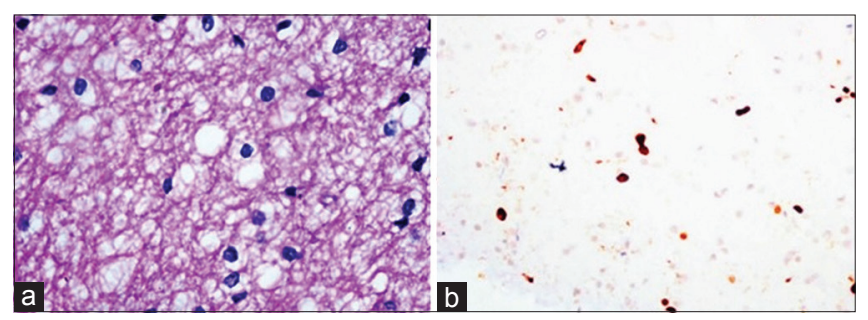

Figure 1: (a and b) Grade II astrocytoma ( $\mathrm{H}$ and $\mathrm{E}, \times 200)$; low proliferative index $(\mathrm{Ki}-67, \times 200)$

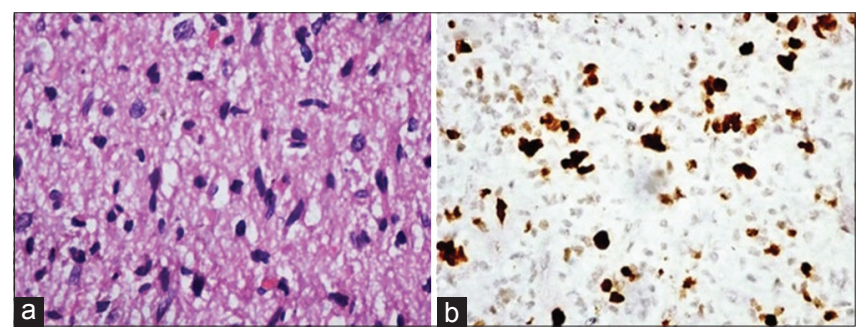

Figure 2: $(\mathrm{a}$ and $b)$ Grade III astrocytoma $(\mathrm{H}$ and $\mathrm{E}, \times 200)$; tumor cells showing intermediate proliferation, $(\mathrm{Ki}-67, \times 200)$

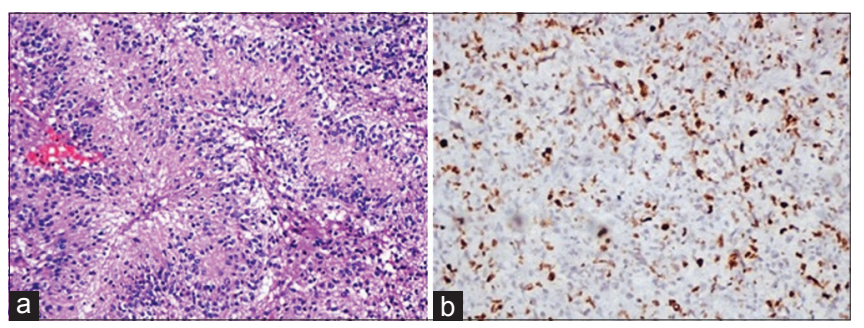

Figure 3: ( $a$ and $b$ ) Grade IV astrocytoma ( $\mathrm{H}$ and E, $\times 200)$; tumor cells showing high proliferation (Ki-67, ×200)

The present study showed a statistically significant difference between Grade II/III and Grade II/IV tumors. The measure of mitotic activity is very important in differentiating Grade II from Grade III astrocytomas, especially in stereotactic biopsies and in cases where the morphology is equivocal, thus indicating the need to measure the proliferative activity along with the $\mathrm{WHO}$ criteria for the diagnosis of these tumors. However, no statistically significant difference was found in Ki-67 LI between Grade III and Grade IV tumors implying that histopathology plays an important role in grading these tumors.

In the present study, immunohistochemical results of three cases of astrocytomas did not show concordance with histopathological grade. Three cases (two cases of anaplastic astrocytomas and one case of gemistocytic astrocytoma Grade III) were histopathologically diagnosed as Grade II astrocytomas, which on Ki-67 immunostaining showed higher LI consistent with Grade III astrocytoma. This implies that histological typing may under- or over-rate the actual biological behavior of astrocytomas. 
The variations in the Ki-67 LI in various studies can be attributed to many factors such as fixative used, immunohistochemical procedures, especially antigen retrieval and interpretation of immunostaining. A low Ki-67 LI in high-grade astrocytoma could result from faulty sampling techniques and heterogeneity of the tumor. Retrieval of antigen can be better with hydrated autoclave treatment than with microwave treatment, which can result in higher Ki-67 LI possibly resulting from successful denaturation of formalin-fixed antigens. ${ }^{[14,20]}$

This study highlights that all histological low-grade astrocytomas may not actually be biologically indolent and it is in this particular subset of patients, IHC scores over histopathological grading. This category of patients with biologically aggressive behavior merits close clinical follow-up and serial neuroimaging to detect early recurrence and malignant transformation.

\section{Conclusion}

The present study demonstrates that there is a highly significant correlation between Ki-67 LI and histopathological grading of astrocytomas. There was a gradual increase in the Ki-67 LI with an increase in tumor grade, though it varied from the proposed cut off values substantially within the grades. However, there was a statistically significant difference in between low-grade (Grade II) and high-grade (Grade III and IV) astrocytomas.

Furthermore, there was an overlap of Ki-67 LIs between the different grades. It was seen that the values of the glioblastoma group could be as low as those for Grade II tumors, indicating that Ki-67 LI cannot be used alone as a diagnostic measure.

Ki-67 should be used judiciously and in combination with other variables such as radiography, clinical status, and established histopathological features of anaplasia. $\mathrm{Ki}-67 \mathrm{LI}$ is a very useful adjunct to the histopathological diagnosis and can be of great help in situations where there is a lack of correlation between histological diagnosis and clinical parameters.

Our study emphasizes that Ki-67 LI is not dependent on factors such as age and sex although the average level of Ki-67 LI varies considerably in the different grades of astrocytomas.

\section{Financial support and sponsorship}

Nil.

\section{Conflicts of interest}

There are no conflicts of interest.

\section{References}

1. Lind-Landström T, Habberstad AH, Sundstrøm S, Torp SH. Prognostic value of histological features in diffuse astrocytomas WHO grade II. Int J Clin Exp Pathol 2012;5:152-8.

2. Ambroise MM, Khosla C, Ghosh M, Mallikarjuna VS, Annapurneswari S. Practical value of MIB-1 index in predicting behavior of astrocytomas. Indian J Pathol Microbiol 2011;54:520-5.

3. Louis DN, Ohgaki H, Wiestler OD, Cavenee WK, Burger PC, Jouvet A, et al. The 2007 WHO classification of tumours of the central nervous system. Acta Neuropathol 2007;114:97-109.

4. Johannessen AL, Torp SH. The clinical value of Ki-67/MIB-1 labeling index in human astrocytomas. Pathol Oncol Res 2006;12:143-7.

5. Malhan P, Husain N, Bhalla S, Gupta RK, Husain M. Proliferating cell nuclear antigen, p53 and micro vessel density: Grade II vs. Grade III astrocytoma. Indian J Pathol Microbiol 2010;53:20-3.

6. Rathi KR, Radotra BD, Khosla VK. Proliferative index in astrocytic tumours. Indian J Pathol Microbiol 2007;50:754-8.

7. Giannini C, Scheithauer BW, Burger PC, Christensen MR, Wollan PC, Sebo TJ, et al. Cellular proliferation in pilocytic and diffuse astrocytomas. J Neuropathol Exp Neurol 1999;58:46-53.

8. Sharma MC, Ralte AM, Gaekwad S, Santosh V, Shankar SK, Sarkar C. Subependymal giant cell astrocytoma - A clinicopathological study of 23 cases with special emphasis on histogenesis. Pathol Oncol Res 2004;10:219-24.

9. Ralte AM, Sharma MC, Karak AK, Mehta VS, Sarkar C. Clinicopathological features, MIB-1 labeling index and apoptotic index in recurrent astrocytic tumors. Pathol Oncol Res 2001;7:267-78.

10. Torp SH. Diagnostic and prognostic role of Ki67 immunostaining in human astrocytomas using four different antibodies. Clin Neuropathol 2002;21:252-7.

11. Neder L, Colli BO, Machado HR, Carlotti CG Jr., Santos AC, Chimelli L. MIB-1 labeling index in astrocytic tumors - A clinicopathologic study. Clin Neuropathol 2004;23:262-70.

12. Karamitopoulou E, Perentes E, Diamantis I, Maraziotis T. Ki-67 immunoreactivity in human central nervous system tumors: A study with MIB 1 monoclonal antibody on archival material. Acta Neuropathol 1994;87:47-54.

13. Khalid H, Shibata S, Kishikawa M, Yasunaga A, Iseki M, Hiura T. Immunohistochemical analysis of progesterone receptor and $\mathrm{Ki}-67$ labeling index in astrocytic tumors. Cancer 1997;80:2133-40.

14. Wakimoto H, Aoyagi M, Nakayama T, Nagashima G, Yamamoto S, Tamaki M, et al. Prognostic significance of Ki-67 labeling indices obtained using MIB-1 monoclonal antibody in patients with supratentorial astrocytomas. Cancer 1996;77:373-80.

15. Di X, Nishizaki'T, Harada K, Kajiwara K, Nakayama H, Ito H. Proliferative potentials of glioma cells and vascular components determined with monoclonal antibody MIB-1. J Exp Clin Cancer Res 1997;16:389-94.

16. Hsu DW, Louis DN, Efird JT, Hedley-Whyte ET. Use of MIB-1 (Ki-67) immunoreactivity in differentiating grade II and grade III gliomas. J Neuropathol Exp Neurol 1997;56:857-65.

17. Avninder S, Sharma MC, Deb P, Mehta VS, Karak AK, Mahapatra AK, et al. Gemistocytic astrocytomas: Histomorphology, proliferative potential and genetic alterations - A study of 32 cases. J Neurooncol 2006;78:123-7.

18. Shaffrey ME, Farace E, Schiff D, Larner JM, Mut M, Lopes MB. The $\mathrm{Ki}$-67 labeling index as a prognostic factor in Grade II oligoastrocytomas. J Neurosurg 2005;102:1033-9.

19. Perry A, Aldape KD, George DH, Burger PC. Small cell astrocytoma: An aggressive variant that is clinicopathologically and genetically distinct from anaplastic oligodendroglioma. Cancer 2004;101:2318-26.

20. Bouvier-Labit C, Chinot O, Ochi C, Gambarelli D, Dufour H, Figarella-Branger D. Prognostic significance of Ki67, p53 and epidermal growth factor receptor immunostaining in human glioblastomas. Neuropathol Appl Neurobiol 1998;24:381-8. 